\section{Strength of Metals}

IN a recent letter under the above title, H. Lipson and A. R. Stokes ${ }^{1}$ suggested that in my work on the subject I have not made sufficient mention of the contributions of themselves and co-workers to the interpretation of X-ray line-broadening from deformed metals.

I have not done so before because all their published work has been on metals which have been deformed by the rough-and-ready process of filing, and has been confined to the examination of the irreg. ular filings so produced. When an engineer thinks of the problem of the deformation and strength of metals, he does so in terms of solid metal systematic. ally deformed by recognized mechanical tests or by standard modes of fabrication. Therefore, as a serious contribution to the problem I have regarded their results as irrelevant, and their assumption that the findings on filings can be applied unmodified to normal metal as unsound.

For the same reason I have not before considered it necessary to comment on their further contention that the line-broadening as interpreted by them is related to the yield strength of metals. It is well known that the yield and ultimate strength of metals depend greatly on the method of deformation and testing. It is sufficient of a problem to understand the effects of normal tests without adding the somewhat unreal case of metal filings.

For curiosity we have compared the X-ray effects from filings and solid metal and find no systematic relation. This observation is referred to in fuller papers on the wider subject which have recently been published by the Institute of Metals ${ }^{2,3}$. The papers are still open for discussion, and if Dr. Lipson and Dr. Stokes wish to make a contribution I should be pleased to comment further.

\section{W. A. WooD}

Baillieu Laboratory,

University, Melbourne.

${ }^{1}$ Lipson, H., and Stokes, A. R., Nature, 163, 871 (1949).

2 Wood, W. A., and Rachinger, W. A., J. Inst. Metals (March 1949). ${ }^{3}$ Wilms, G. R., and Wood, W. A., J. Inst. Metals (April 1949).

The main difierence between Dr. Wood and ourselves seems to lie in our interpretation of the functions associated with a physicist. We believe that a physicist should be concerned with splitting a problem into its essentials and dealing with them separately, whereas the engineer should deal with immediate problems in their entirety. One is familiar with the accusation that physicists are too far removed from reality to be of practical use; one does not expect this accusation to be repeated by another physicist such as Dr. Wood.

Our experiments on filings were conducted in this light. We believe that filings are simpler to deal with theoretically than massive metal, because any effects on them are more likely to average out ; as a parallel, the dynamics of a large number of particles can be handled although the three-body problem is insoluble. We do not claim that our results are complete, but merely that they provide important evidence in considering the more complicated state of affairs in massive metal.

We are aware of the recent papers to which Dr. Wood refers, but to undertake an answer to them would necessitate further experimental work for which we are not equipped. We would, however, refer to one major point : we cannot accept the contention that it is possible for an appreciable volume of metal, plastically deformed, to be in a state of uniform stress.

Finally, we would point out that we did not direct attention only to our own work, as Dr. Wood states; two other schools were included in the references we gave. Moreover, so far as we are aware, no other workers have found it possible to support Dr. Wood's results. We recognize, however, that no single type of experiment can be conclusive, and we would suggest that the interests of the subject might be better served by the exploration of other methods of experimentation, such as micro-techniques, rather than by the measurement of line-breadths only. Dr. Wood's own results, published in $1932^{1}$, illustrate perfectly how deceptive the measurement of one or two lines on a back-reflexion photograph can be.

Department of Physics,

\section{H. LIPSON}

College of Technology, Manchester.

Wheatstone Physics Laboratory,

A. R. STokfs

King's College,

London, W.C.2.

${ }^{1}$ Nature, 129, 760 (1932).

\section{Origin of Hygroscopicity of Jute}

JUTE is reported ${ }^{1}$ to be characterized by high values of moisture adsorption and heat of wetting. From a study of the adsorption isotherms and heats of wetting of a series of cellulose fibres including cotton, ramie and rayons, Hermans ${ }^{2}$ has concluded that greater hygroscopicity of rayons relative to pure cellulosic native fibres is due to the presence of a correspondingly greater quantity of amorphous cellulose. The results of his X-ray method ${ }^{3}$ of quantitative evaluation of the crystalline fraction check well with his findings from adsorption isotherms and heats of wetting.

\begin{tabular}{|c|c|c|}
\hline \multirow{2}{*}{ Relative humidity (per cent) } & \multicolumn{2}{|c|}{ Sorption ratio } \\
\cline { 2 - 3 } & Adsorption & Desorption \\
\hline 15 & $1 \cdot 60$ & $1 \cdot 40$ \\
30 & $1 \cdot 70$ & 1.45 \\
45 & $1 \cdot 60$ & 1.50 \\
60 & 1.65 & 1.57 \\
75 & $1 \cdot 60$ & 1.55 \\
85 & $1 \cdot 60$ \\
\hline Average & \\
\hline
\end{tabular}

The average sorption ratio of jute calculated from the published results for cotton 4 and jute (see table) is $1 \cdot 60$. The heat of absorption of jute at zero regain calculated from the heat of wetting-regain curve by extrapolation is $228 \mathrm{cal} / \mathrm{gm}$. water, which is nearly the same value as reported by $\operatorname{Rees}^{5}$ for cotton, rayon, etc. It appears, therefore, that the greater hygroscopicity of jute can be attributed to the capacity factor, on account of a greater quantity of amorphous constituents in polyuronides, xylan, hexosan and lignin in the fibre. If this view is correct, the ratio of the integral heats of wetting at zero regain of cotton and jute should be equal to the 